\title{
Melatonin is responsible for rice resistance to rice stripe virus infection through a nitric oxide-dependent pathway
}

Rongfei Lu ${ }^{1,2+}$, Zhiyang $\mathrm{Liu}^{2+}$, Yudong Shao ${ }^{1,2}$, Feng Sun ${ }^{2}$, Yali Zhang ${ }^{3}$, Jin Cui ${ }^{1}$, Yijun Zhou ${ }^{2}$, Wenbiao Shen ${ }^{{ }^{*}}$ and Tong Zhou $2,4,5^{*}$ (D)

\begin{abstract}
Rice stripe virus (RSV) causes one of the most important rice virus diseases of plants in East Asia. However, the molecular mechanisms controlling rice resistance to RSV infection are largely unknown. Recently, several studies presented a novel model that melatonin (MT) and nitric oxide (NO) participate in the plant-pathogen interaction in a synergetic manner. In this study, there was a difference in MT content between two rice varieties that correlated with one being susceptible and one being resistant to RSV, which suggested that MT is related to RSV resistance. In addition, a test with two NO biosynthesis inhibitors revealed that NO inhibitor were able to increase the disease incidence of RSV. A pharmacological experiment with exogenous MT and NO showed that increased MT and NO in the MT-pretreated plants led to lower disease incidences; however, only NO increased in a NO-releasing reagent [sodium nitroprusside (SNP)] pretreated plants. The expressions level of OsPR1b and OsWRKY 45 were significantly induced by MT and NO. These results suggest that rice resistance to RSV can be improved by increased MT through a NO-dependent pathway.
\end{abstract}

Keywords: Melatonin, Nitric oxide, Rice stripe virus, Rice

\section{Introduction}

Rice stripe virus (RSV), a typical member of the genus Tenuvirus, is transmitted mainly by the small brown planthopper (Laodelphax striatellus) (SBPH) in a persistent and propagative manner, and can infect many crops, such as rice, wheat, and several other gramineous plants, resulting in a severe loss of grain [1]; in addition, Sun et al. reported that the virus can also infect Arabidopsis thaliana [2]. The infected plants exhibit typical symptoms, such as alternating yellow and green stripes, curly and drooping leaves, and necrotic lesions [3]. Previous reports indicated that the plant chloroplasts were damaged by excessive accumulation of starch, which was triggered by RSV infection, resulting in alternating

\footnotetext{
* Correspondence: wbshenh@njau.edu.cn; zhoutong@jaas.ac.cn

${ }^{\dagger}$ Rongfei Lu and Zhiyang Liu contributed equally to this work.

${ }^{1}$ College of Life Sciences, Laboratory Center of Life Sciences, Nanjing

Agricultural University, Nanjing 210095, China

${ }^{2}$ Key Laboratory of Food Quality and Safety, Institute of Plant Protection,

Jiangsu Academy of Agricultural Sciences, Nanjing 210014, Jiangsu Province,

China

Full list of author information is available at the end of the article
}

yellow and green stripes [4]. Melatonin (N-acetyl-5methoxytryptamine, MT), an amine hormone that was isolated and identified from the pineal gland in the late 1950s, has been revealed to possess a broad spectrum of biological functions in both animals and plants in many studies [5-9]. In particular, MT has been considered as a therapeutic indole for combating viral diseases, such as SARS (severe acute respiratory syndrome) and WNV (West Nile virus) [10]. Consistent with the benefits of MT in immunology and medicine in animals, MT can also induce plant resistance to pathogens, such as Pseudomonas syringae DC3000, Alternaria spp., and Fusarium spp. $[11,12]$. However, the detailed mechanisms of MT -triggered plant innate immunity to viruses are elusive. Recently, several studies also indicated the potential involvement of a gaseous signal molecule or plant hormone in the defense response triggered by MT. For example, it was reported that nitric oxide (NO) induced by MT is responsible for disease resistance to Pst DC3000 infection in Arabidopsis [13]. Indeed, the vital role of $\mathrm{NO}$ in plant-pathogen interactions has been

(C) The Author(s). 2019 Open Access This article is distributed under the terms of the Creative Commons Attribution 4.0 International License (http://creativecommons.org/licenses/by/4.0/), which permits unrestricted use, distribution, and 
demonstrated by many studies [14-18]. NO can be produced through the nitrate/nitrite-dependent pathway that is known to be catalyzed by nitrate reductase (NR) [19] and through the $\mathrm{L}_{\mathrm{L}}$-arginine-dependent pathway that is known to be catalyzed by a mammalian NO synthase (NOS)-like enzyme [20, 21]. Further, Lee et al. found that the disease resistance induced by MT correlates with plant hormones in Pst DC3000 [11, 22]. Thus, these studies may provide a potential mechanism for the effect of MT and NO in plant against other pathogen infections.

In this study, the endogenous MT level was monitored and quantified in rice after RSV infection. Further, the link among MT, NO and resistance genes was investigated.

\section{Materials and methods Chemicals}

Unless otherwise stated, all chemicals used in this study were purchased from Sigma-Aldrich (Sigma-Aldrich, St Louis, MO, USA). In this study, sodium nitroprusside (SNP) was used as a NO-releasing reagent, diluted to $100 \mu \mathrm{M}$ in water, and it was applied to soil in pots $(9 \mathrm{~cm}$ in diameter and $14 \mathrm{~cm}$ tall, $25 \mathrm{~mL} /$ pot) with rice seedlings. The old SNP solution was obtained as a negative control by maintaining a $100 \mu \mathrm{M}$ SNP solution for at least 5 days in the light in an open tube to eliminate NO as described [23, 24]. 2-(4-carboxyphenyl)-4, 4, 5, 5tetramethylimidazoline-1-oxyl-3-oxide (cPTIO), a NO specific scavenger, was diluted to $100 \mu \mathrm{M}$ in water prior to use. In addition, $200 \mu \mathrm{M}$ NG-nitro-L-Arg methyl ester hydrochloride (L-NAME; a nitric oxide synthase (NOS) inhibitor) and $200 \mu \mathrm{M}$ tungstate (a nitrate reductase (NR) inhibitor), were applied.

\section{Plant materials, virus isolates and inoculation assay}

Rice (Oryza sativa L. cv.) seeds of Nipponbare and Zhendao 88, which are susceptible and resistant to RSV, respectively, were both obtained from the Jiangsu Academy of Agricultural Science (JAAS), Jiangsu, China. The viruliferous and virus-free $\mathrm{SBPH}$ used throughout the experiment were fed at the Institute of Plant Protection in JAAS. Young instar nymphs of SBPH were maintained on healthy rice seedlings (Oryza sativa L. c v. Wuyujing No. 3) in an insect-rearing room at $25^{\circ} \mathrm{C}$ until infestation. The rate of viruliferous $\mathrm{SBPHs}$ in the virusacquiring group was detected using a Dot enzyme-linked immunosorbent assay (Dot-ELISA) according to Zhou et al. [25].

The soil of 14-day-old rice seedlings was kept as dry as much as possible, which was followed by various treatments and placement in darkness for $12 \mathrm{~h}$. Then, rice seedlings were inoculated with viruliferous nymphs according to the inoculation method from Zhou et al. for 3 days [26]. Subsequently, all insects were removed from the plants, which were transferred to the soil in the cement pool of greenhouse in the green house. In addition, virus-free SBPHs were used for the control group.

\section{The analysis of disease incidence of RSV}

Thirty rice seedlings of each cultivar were tested for disease incidence experiment. At 30 days post inoculation, the amount of symptomatic plants was counted, and the disease incidence of RSV is the percentage of the symptomatic plants in total 30 plants.

\section{Quantitative analysis of melatonin}

Approximately $100 \mathrm{mg}$ rice leaves inoculated with RSV were harvested and immediately ground under liquid nitrogen; the powder was extracted in $900 \mu \mathrm{L}$ of $10 \mathrm{mM}$ PBS (phosphate buffered saline) buffer ( $\mathrm{pH} 7.2)$ and centrifuged at $12,000 \mathrm{~g}$ for $10 \mathrm{~min}$, the supernatant was prepared for assay. A melatonin detection ELISA kit, purchased from Jiangsu Bao Lai Biological Technology Co., Ltd. China, was used to quantify the endogenous melatonin, following the manufacturer's instructions. All experiments were repeated at least three times.

\section{Quantification of NO content by laser scanning confocal microscopy}

Cross sections ( $3 \mathrm{~mm}$ thick) were cut from stems of viruliferous or nonviruliferous SBPH-inoculated rice plants, infiltrated with an NO fluorescent probe $(10 \mu \mathrm{M} 4$ amino-5-methyl-amino-2', $7^{\prime}$-di-fluorofluorescein diacetate [DAF-FM DA]) diluted in a $20 \mathrm{mM}$ Hepes- $\mathrm{NaOH}$ buffer, $\mathrm{pH} 7.2$, followed by $15 \mathrm{~min}$ incubation in the dark $[27,28]$.

After thoroughly rinsing in the Hepes- $\mathrm{NaOH}$ buffer, the sections were examined, imaged, and processed using a Zeiss LSM 710 confocal laser scanning microscope equipped with ZEN software (Carl Zeiss, Oberkochen, Germany). The excitation wavelength was set at $488 \mathrm{~nm}$, and the emission wavelength was set at 500 $530 \mathrm{~nm}$. More than 10 rice plants were analyzed for each treatment. The average of fluorescence intensity for each treatment was shown in the graph.

\section{RNA isolation and quantitative real-time PCR}

Total RNAs from $100 \mathrm{mg}$ of rice leaves were extracted with Trizol reagent (Invitrogen, Gaithersburg, MD, USA) according to the manufacturer's instructions, and then they were dissolved in DNase-treated distilled water. The concentration and quality of isolated RNA were determined using a NanoDrop 2000 spectrophotometer (Thermo Fisher Scientific, Wilmington, DE, USA). Next, cDNA was synthesized from $1 \mu \mathrm{g}$ of total RNA using an oligo (dT) primer and MMLV reverse transcriptase (BioTeke, Beijing, China). RT-qPCR was 
performed using SsoFastTM Eva Green ${ }^{\circ}$ Supermix (BioRad, Shanghai, China) with the Bio-Rad iQ5 RT-qPCR system. Multiple internal reference genes, $U B Q 10$ and GAPDH, were used in this assay [29]. All primers used in this experiment are shown in Additional file 1: Table S1. The $2^{-\Delta \Delta C t}$ method [30] was used to calculate relative expression levels.

\section{Statistical analysis}

All experiments were repeated three independent times and achieved similar results. Data represent the means \pm SD of triplicate measurements. Statistical analysis was performed using SPSS 18.0 and Duncan's multiple tests, and $P<0.05$ was considered as significant different and indicated with a different letter or asterisk.

\section{Results}

\section{Zhendao 88 is resistant to RSV infection}

To detect the relationship of MT and NO with resistance to RSV, we first verified the different phenotypes presented by Nipponbare and Zhendao 88, which were susceptible and resistant to RSV, respectively. As shown in Fig. 1, compared to the control group, Nipponbare had more severe symptoms than Zhendao 88 (Fig. 1a). The expression level of $C P$ in the two rice varieties was assayed after RSV infection. Quantitative RT-PCR using
RSV CP specific primers showed that RSV RNA accumulated similar in both RSV-inoculated Zhendao 88 and Nipponbare plants at 7, 14 and 21 dpi, but RSV RNA accumulated in Zhendao 88 plants was much lower than in Nipponbare plants at $30 \mathrm{dpi}$ the expression level of $C P$ was significantly higher in Nipponbare than Zhendao 88 at 28 and 35 dpi (Fig. 1b). Consistently, approximately $85 \%$ of the RSV-inoculated Nipponbare plants showed virus symptoms, while only approximately $10 \%$ of the RSV-inoculated Zhendao 88 plants showed virus symptoms (Fig. 1c). Thus, these two rice cultivars were used for further analysis.

\section{The endogenous MT content increased in resistant cultivar Zhendao 88 after RSV infection}

To explore whether MT is responsible to rice resistance to RSV, we assayed the difference in MT content between Nipponbare and Zhendao 88, which were demonstrated to be susceptible and resistant to RSV, respectively, in Fig. 1. The time-course experiment showed that the relative MT content in Zhendao 88 was significantly higher than Nipponbare (Fig. 2a). In addition, in the inhibitor assay with L-NAME and Tungstate, NOS and NR inhibitors, disease incidence increased significantly with NOS inhibitor and inhibitors combination, indicating that NO was also responsible for the resistance to RSV infection (Fig.
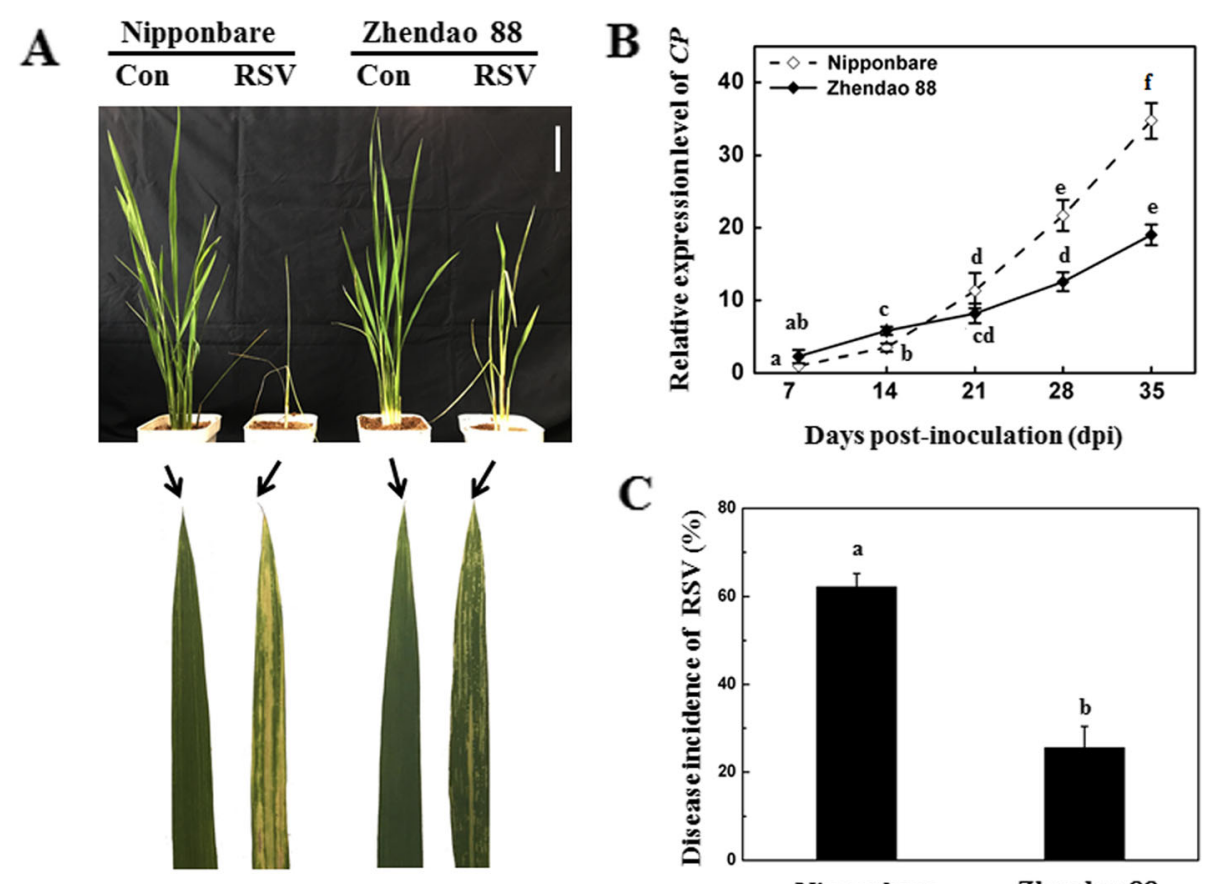

C

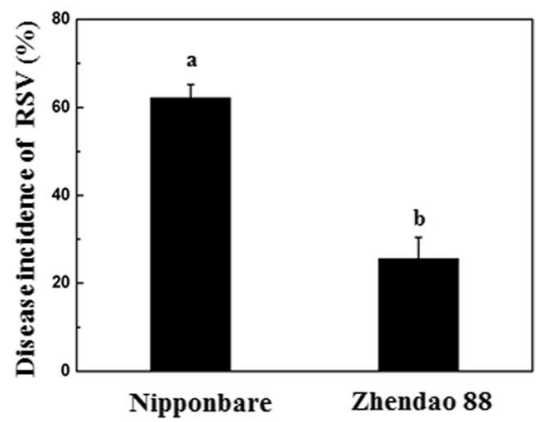

Fig. 1 Different phenotypes of Nipponbare (susceptible) and Zhendao 88 (resistant) inoculated with RSV. a Symptoms of the two varieties at 28 dpi. The lower panel of (a) shows the symptomatic leaves of the representative plants. Bars $=5 \mathrm{~cm}$. $\mathbf{b}$ Relative expression of RSV CP in the two varieties at 7, 14, 21, 28 and 35 dpi. c Disease incidences of the two varieties at $30 \mathrm{dpi}$. Thirty plants were used for each treatment in the experiment of disease incidence. All the experiments were repeated three times, and similar results were obtained. The data represent the means \pm SD of triplicate measurements. Different letters represent significantly difference at $P<0.05$ according to Duncan's multiple tests 
A

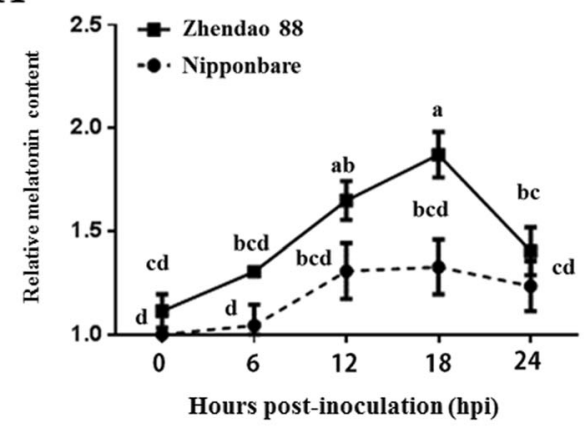

C

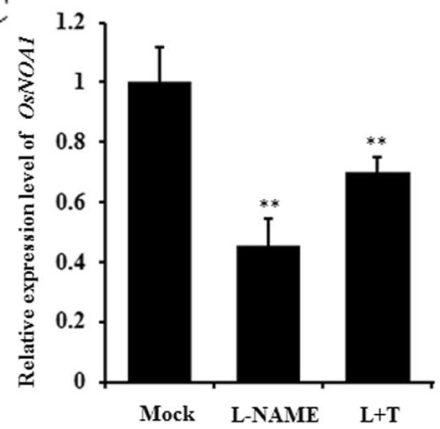

B

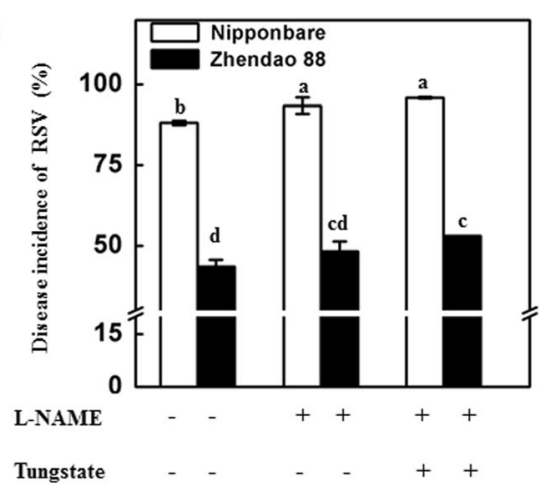

Fig. 2 The endogenous MT content in rice plants after RSV infection. a Relative endogenous MT level of the two varieties in response to RSV pathogen infection within $24 \mathrm{~h}$. The value of the control group was set at 1.0. b Disease incidence of the two varieties pretreated with $200 \mu \mathrm{M} \mathrm{L-}$ NAME (NOS inhibitor), or the combination of treatments with $200 \mu \mathrm{M}$ L-NAME and $200 \mu \mathrm{M}$ Tungstate (NR inhibitor). c Effect of inhibitors on the expressions of OsNOA1 in Nipponbare plants under normal conditions. Mock: water treated. L-NAME: $200 \mu \mu M$ L-NAME treated. L+ T: $200 \mu M M$ LNAME and $200 \mu \mathrm{M}$ Tungstate together. All the experiments were: repeated three times, and similar results were obtained. The data represent the means \pm SD of triplicate measurements. Different letters or asterisks represent significantly difference at $P<0.05$ according to Duncan's multiple tests

2b). Under normal condition, qRT-PCR results showed that the relative expression level of $O S N O A 1$, which was shown to be related to the NOS-dependent pathway [31, 32], was significantly lower in the L-NAME-treated and Tungstate plus L-NAME-treated plants (Fig. 2c). The results suggested that NO content indeed decreased following applications of these two inhibitors. All the results in Fig. 2 were a preliminary indication of the positive role of $\mathrm{MT}$ and NO in the response of rice to RSV infection.

\section{The resistance to RSV can be improved by increased MT through a NO-dependent pathway}

To further analyze the function of MT and NO in the response of rice to virus, Nipponbare plants were pretreated with exogenous MT and NO. We initially screened the optimum concentration of MT and SNP, a specific NO donor. The results showed that MT and SNP could both reduce the disease incidence in a concentration dependent manner, with the largest effects at $10 \mu \mathrm{M}$ of MT and $100 \mu \mathrm{M}$ of SNP, resulting in 30.00 and $25.78 \%$ disease incidence reduction, respectively (Fig. 3a and b). Thus, $10 \mu \mathrm{M}$ MT and $100 \mu \mathrm{M}$ SNP were selected for the follow-up experiment. Moreover, $100 \mu \mathrm{M}$ OldSNP and $100 \mu \mathrm{M}$ cPTIO were also pretreated in the inoculation experiment. As shown in Fig. 3c, compared to the water-treated group, Old-SNP treatment failed to reduce the disease incidence as SNP did, and cPTIO can exacerbate disease, which indicated that $\mathrm{NO}$ may play a vital role in the rice response to RSV infection. In addition, cPTIO could eliminate the MT positive effect on the disease incidence, which suggested that NO serves as a mediator in MT triggered rice resistance to RSV infection. Overall, we speculated that MT and NO can promote rice resistance to RSV, and NO functions downstream of MT in this pathway.

To further detect the participation of MT and NO in rice-virus interactions, we assayed the production of MT and NO in rice plants after RSV infection firstly. The result showed that MT and NO production were accumulated higher in RSV-inoculated rice plants than water-treated virus-free inoculated plants, which indicated that the MT and NO production might be induced in rice-RSV interactions. Results of the MT and NO treatments experiments showed that MT-treated rice plants accumulated about more MT and NO compared with water-treated virus-free inoculated plants, while SNP-treated rice plants accumulated more NO but not MT (Fig. 3d and e). Taken all results in Figs. 3 together, it demonstrated that MT and NO 

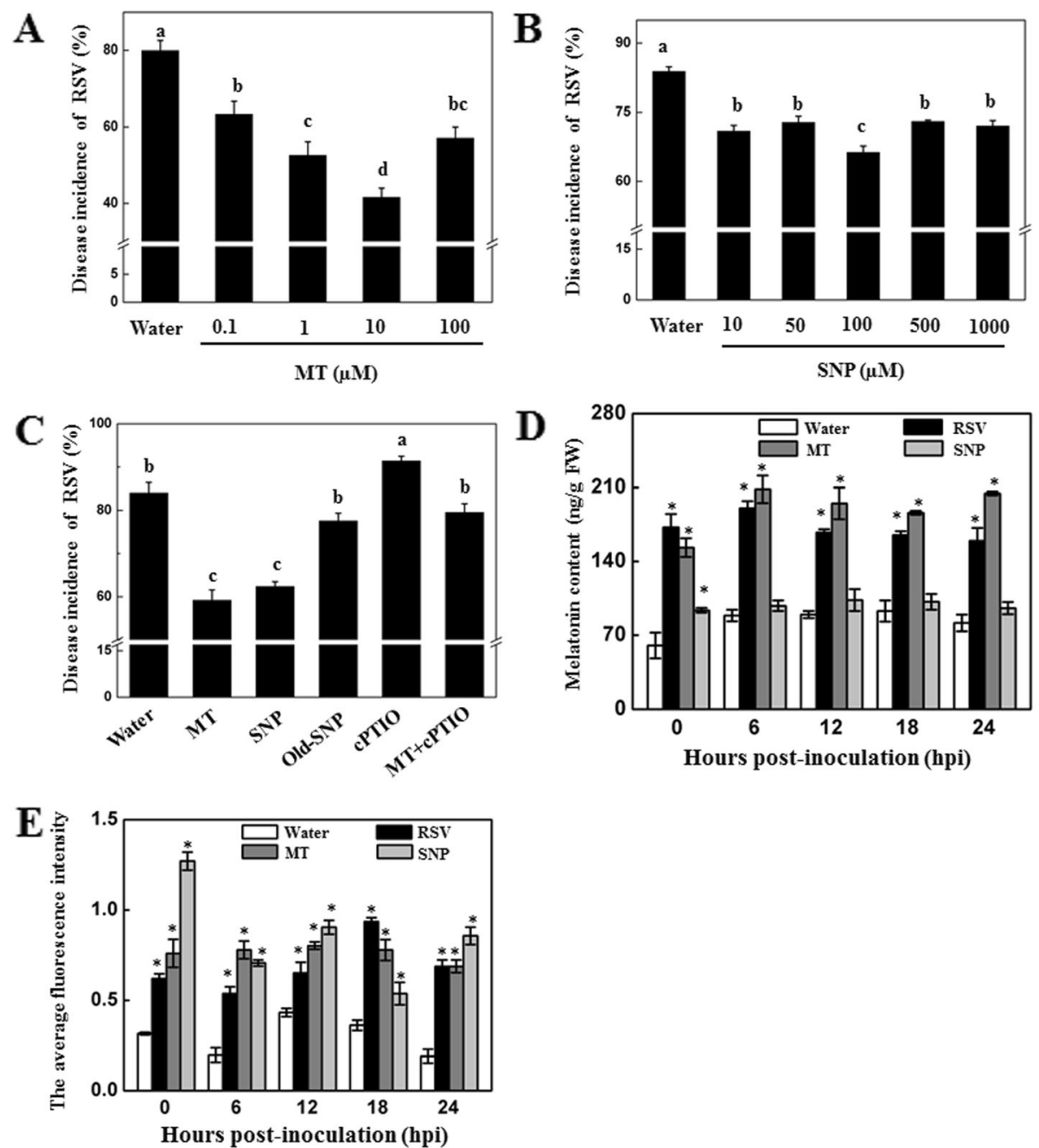

Fig. 3 The resistance to RSV can be improved by the increased production of MT and NO. The high disease incidence of Nipponbare inoculated with RSV can be reduced by exogenous MT and NO. The disease incidence of Nipponbare was recorded at 30 dpi pretreated with: (a) The disease incidence of Nipponbare inoculated with RSV pretreated with four different concentrations of MT (0.1, 1, 10 and $100 \mu \mathrm{M})$ or (b) with five different concentrations of SNP $(10,50,100,500$ and $1000 \mu \mathrm{M})$ for $12 \mathrm{~h}$ and inoculated with viruliferous for $3 \mathrm{~d}$. c The disease incidence of Nipponbare pretreated with deionized water, $10 \mu \mathrm{M}$ MT, $100 \mu \mathrm{M}$ SNP, $100 \mu \mathrm{M}$ Old-SNP, $100 \mu \mathrm{M}$ CPTIO and $10 \mu \mathrm{M}$ MT + $100 \mu \mathrm{M}$ CPTIO for $12 \mathrm{~h}$ and then inoculation with RSV for $3 \mathrm{~d}$. All data were recorded at $30 \mathrm{dpi}$. Thirty plants were used for each treatment in the experiment of disease incidence. $\mathbf{d}$ and (e) Nipponbare plant treated with virus-free SBPH, RSV (viruliferous SBPH), $10 \mu \mathrm{M}$ MT or $50 \mu \mathrm{M}$ SNP. The rice plants were inoculated by virus-free SBPH or viruliferous SBPH for 3 days, then all SBPHs were removed from the plants, the time point when SBPHs were removed was set as $0 \mathrm{hpi}$. After $0,6,12,18$ and $24 \mathrm{~h}$, plants were taken immediately for the melatonin or NO assays. All the experiments were repeated three times, and similar results were obtained. The data represent the means \pm SD of triplicate measurements. Different letters or asterisks represent significantly difference at $P<0.05$ according to Duncan's multiple tests

can respond to RSV infection, and the effect of MT on rice resistance to RSV is through a NO-dependent pathway.

\section{Expression patterns of OsPR 16 and OsWRKY 45 after various treatments}

Since OsWRKY 45 was reported to play an essential role in rice blast resistance [33] and it is well-known that induced expression of PR genes is able to enhance the plant's disease-resistance [34], the expression levels of OsPR $1 b$ and OsWRKY 45 in rice plants with various treatments were analyzed at $18 \mathrm{hpi}$ using quantitative real-time
PCR. Compared to the water-treated group, the two genes were induced by both MT and SNP treatment and suppressed by cPTIO significantly after RSV infection, while the expression levels of OsPR1b was also induced by MT and SNP treatments, and OsWRKY 45 was induced by SNP treatment in uninfected plants, although the inducible level is lower than that in RSV infected plants (Fig. 4). The results indicated that OsPR $1 b$ and OsWRKY 45 were induced by RSV infection and OsPR $1 b$ might act downstream of MT and NO signaling pathway, and OsWRKY 45 could be partially downstream of MT and NO signaling 

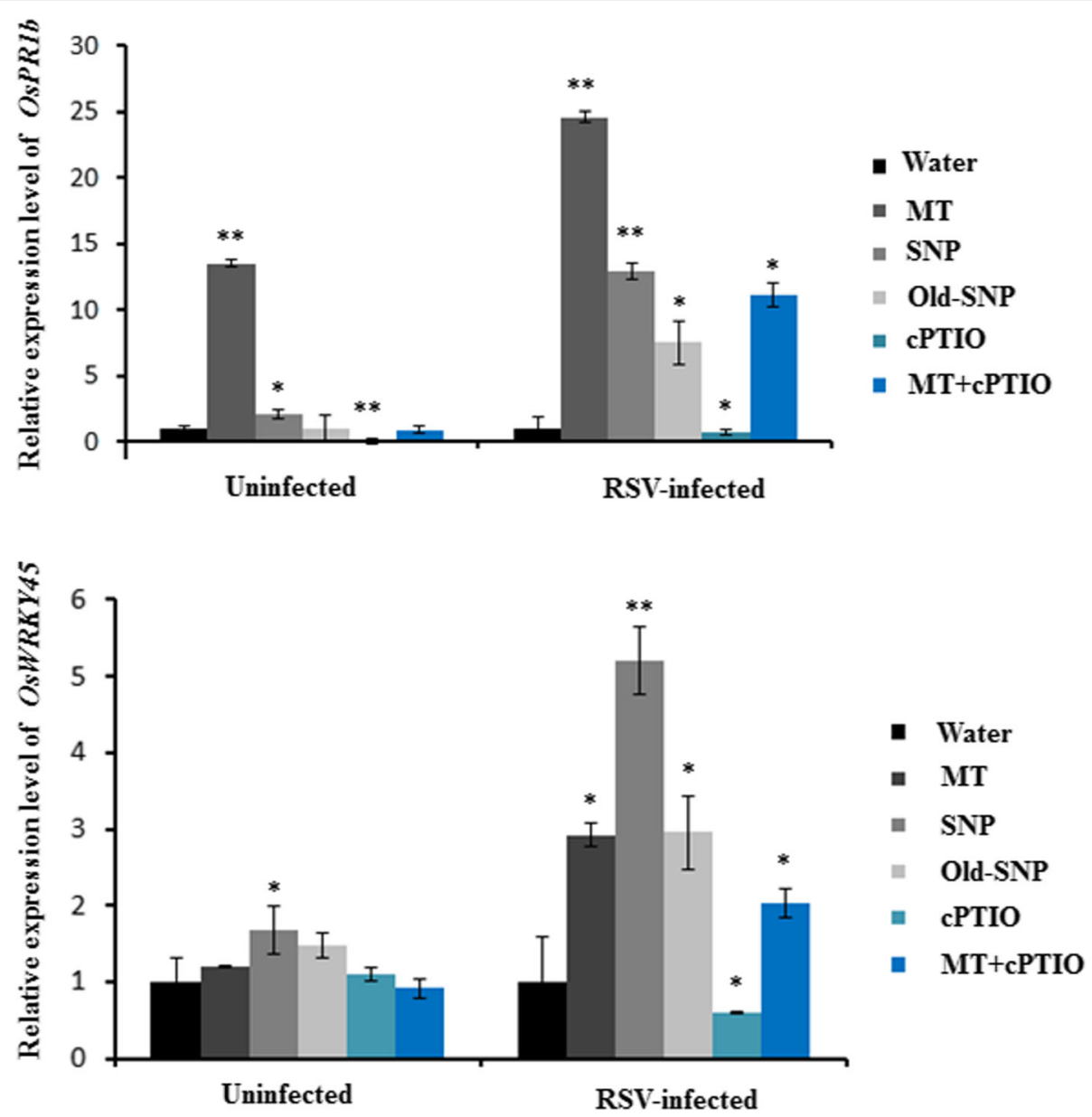

Fig. 4 Transcriptional profiles of OSPR1b and OSWRKY 45. Nipponbare plants were pretreated with different treatments and then were inoculated with virus-free SBPH or RSV (viruliferous SBPH). Total RNA was extracted from rice leaves at $18 \mathrm{hpi}$. The assay was performed by qPCR and normalized against the combination of UBQ 10 and GAPDH. The values of the water-treated group were set at 1.0. All the experiments were repeated three times, and similar results were obtained. The data represent the means \pm SD of triplicate measurements. Different letters or asterisks represent significantly difference at $P<0.05$ according to Duncan's multiple tests

pathway, because of the low expression level after the treatments in uninfected plants. Interesting, Old-SNP and the combination of MT and cPTIO could also up-regulate their expression, although the change was relatively minor; thus, further study is needed to determine whether they function in other pathways.

\section{Discussion}

Because of the lack of effective protection against rice damage caused by RSV infection and the concerns about the safety of transgenic technology, exogenous applications of protective biological treatments may be acceptable and environmentally friendly [35]. Although some studies have shown that MT and NO can improve the resistance of plants to pathogens, their effect on ricevirus interactions remains elusive [11, 13, 36]. Recently, the association between MT and NO in disease resistance has been reported not only in animals but also in plants [13, 37]. Consistent with these studies, we found that NO participates in MT triggered rice resistance to RSV.

The first evidence is that MT can be significantly induced in resistant cultivar in response to RSV infection (Fig. 2a), and accordingly, it correlated with high levels of rice resistance to RSV (Fig. 1c). These results preliminarily suggested the protective role of MT to RSV stress. Secondly, pharmacological experiments with both exogenous MT and NO alleviated the disease incidence of RSV, while application with cPTIO had a negative effect on disease incidence, which indicates that MT and NO can improve rice resistance against the RSV pathogen (Fig. 3). Overall, our results demonstrated the vital role of MT and NO in the rice-RSV interaction.

Strikingly, we found that cPTIO can reverse the positive effect of MT on disease incidence, which led us to deduce that $\mathrm{NO}$ is a mediator in the process of MT 
triggered rice resistance to RSV. Moreover, MT application can significantly enhance NO content level, while a NO donor has no effect on endogenous MT levels (Fig. $3)$. These results further indicated that NO is a downstream signal component of MT triggered rice resistance to RSV, which is consistent with the previous report of Shi et al. on Arabidopsis [13]. However, this mode of action is not always the case, because Pozo et al. and Aydogan et al. both reported the inhibitory effect of MT on biosynthesis of NO $[38,39]$. Thus, the detailed mechanism of the concentration-dependent interaction between MT and NO in plant-pathogen interactions needs to be studied further.

According to the previously article [40], the rice variety Zhendao 88 did carry a resistant allele of STV11, which encoded a sulfotransferase that catalyzes the conversion of salicylic acid into sulphonated SA, confers resistance in RSV. It has also been reported that NO is required for the full function of SA [41], we speculated that the increase of MT in Zhendao 88 could lead more accumulated NO, which might lead to more SA. So sulphonated SA may be increased by the conversion of accumulated SA, which may be the reason why Zhendao 88 is resistant to RSV.

Oryza sativa WRKY transcription factor, OsWRKY 45, was reported to promote systemic acquired resistance (SAR) independent of OsPR1 [33, 42], although not all of the WRKY genes reported are positive during pathogenesis [43]. Therefore, we further assayed the relative expression of OsPR $1 b$ and OsWRKY 45, as shown in Fig. 4; it was demonstrated that both MT and SNP application positively modulated these two genes, which confirmed our results described above.

In conclusion, $\mathrm{MT}$ is responsible for rice resistance to RSV infection by inducing NO. However, not only gas signaling molecules and plant hormones but also other components, such as carbohydrate and alcohols, also participate in the MT triggered plant resistance to pathogen infection [44]. Thus, it may have a broad application prospect on the further in-depth study of MT in plant-pathogen interactions.

\section{Supplementary information}

Supplementary information accompanies this paper at https://doi.org/10. 1186/s12985-019-1228-3.

Additional file 1: Table S1. Primers used for quantitative reverse transcription polymerase chain reaction (qRT-PCR).

\section{Abbreviations}

CP: Coat protein; CPTIO: 2-(4-carboxyphenyl)-4, 4, 5, 5-tetramethylimidazoline1-oxyl-3-oxide; MT: Melatonin; NO: Nitric oxide; NOS: NO synthase; NR: nitrate reductase; RSV: Rice stripe virus; SBPH: Small brown planthopper; SNP: a NOreleasing reagent

\section{Acknowledgements}

We are very grateful to Xiaoling Ye (Jiangsu academy of agricultural sciences, Nanjing, China) for the management of greenhouse.

\section{Authors' contributions}

Conceptualization, WS and TZ; Data curation, RL; Formal analysis, ZL; Methodology, RL and YS; Project administration, YZhou; Resources, YZhang; Supervision, JC, WS and TZ; Validation, ZL, YS and FS; Writing - original draft, RL; Writing - review \& editing,

ZL. All authors read and approved the final manuscript.

\section{Funding}

This work was supported by the National Natural Science Foundation of China (31761143012, 31601704), Six talent peaks project of Jiangsu Province (NY-056), 333 high level talent training project of Jiangsu Province (BRA2018081)

\section{Availability of data and materials}

All data generated or analyzed during this study are included in this published article.

Ethics approval and consent to participate

Not applicable.

\section{Consent for publication}

Not applicable.

\section{Competing interests}

The authors declare that the research was conducted in the absence of any commercial or financial relationships that could be construed as a potential conflict of interest.

\section{Author details}

${ }^{1}$ College of Life Sciences, Laboratory Center of Life Sciences, Nanjing Agricultural University, Nanjing 210095, China. ${ }^{2}$ Key Laboratory of Food Quality and Safety, Institute of Plant Protection, Jiangsu Academy of Agricultural Sciences, Nanjing 210014, Jiangsu Province, China. ${ }^{3}$ College of Resources and Environmental Sciences, Nanjing Agricultural University, Nanjing 210095, China. ${ }^{4}$ School of the Environment and Safety Engineering, Jiangsu University, Zhenjiang 212013, Jiangsu Province, China. ${ }^{5}$ International Rice Research Institute and Jiangsu Academy of Agricultural Sciences Joint Laboratory, Nanjing 210095, China.

Received: 4 June 2019 Accepted: 23 September 2019

Published online: 21 November 2019

\section{References}

1. Milne RG. The Filamentous Plant Viruses. In: Milne RG, editor. The Plant Viruses. New York: Plenum Press; 1988. p. 297-329.

2. Sun F, Yuan X, Zhou T, Fan Y, Zhou Y. Arabidopsis is susceptible to Rice stripe virus infections. J Phytopathol. 2011;159:767-72.

3. Toriyama S. Rice stripe virus: prototype of a new group of viruses that replicate in plants and insects. Microbiol Sci. 1986:3:347.

4. Yamashit S, Doi Y, Yora K. Intracellular appearance of rice stripe virus. Ann Phytopathol Soc Jpn. 1985;51:637-41.

5. Lerner A, Case J, Takahashi Y, Lee TH. Isolation of melatonin, the pineal gland factor that lightens melanocytes. J Amer Chem Soc. 1958;80:10.

6. Arnao MB, Hernándezruiz J. The physiological function of melatonin in plants. Plant Signal Behav. 2006;1:89-95.

7. Reiter R, Tan D, Fuentesbroto L. Melatonin: a multitasking molecule. Prog Brain Res. 2010;181:127-51

8. Liang C, Zheng G, Li W, Wang Y, Hu B, Wang H, et al. Melatonin delays leaf senescence and enhances salt stress tolerance in Rice. J Pineal Res. 2015;59:91.

9. Zhang N, Sun Q, Zhang H, Zhao C, Li L, Chen M. Roles of melatonin in abiotic stress resistance in plants. J Exp Bot. 2015;66:647.

10. Bonilla E, Valero N, Chacín-Bonilla L, Medina-Leendertz SJ. Melatonin and viral infections. J Pineal Res. 2004;36:73-9.

11. Lee $H$, Byeon $Y$, Back K. Melatonin as a signal molecule triggering defense responses against pathogen attack in Arabidopsis and tobacco. J Pineal Res. $2014 ; 57: 262-8$. 
12. Arnao MB, Hernández-Ruiz J. Functions of melatonin in plants. J Pineal Res. 2015;59:133.

13. Shi $H$, Chen $Y$, Tan D, Reiter R, Chan Z, He C. Melatonin induces nitric oxide and the potential mechanisms relate to innate immunity against bacterial pathogen infection in Arabidopsis. J Pineal Res. 2015;59:102-8.

14. James SL. Role of nitric oxide in parasitic infections. Microbiol Rev. 1995;59: 533-47.

15. Akaike T, Maeda H. Nitric oxide and virus infection. Immunology. 2000;101: 300-8.

16. Hong JK, Yun BW, Kang JG, Raja MU, Kwon E, Sorhagen K, et al. Nitric oxide function and signalling in plant disease resistance. J Exp Bot. 2008;59:147-54.

17. Perchepied L, Balagué C, Riou C, Claudel-Renard C, Rivière N, Grezes-Besset $B$, et al. Nitric oxide participates in the complex interplay of defense-related signaling pathways controlling disease resistance to Sclerotinia sclerotiorum in Arabidopsis thaliana. Mol Plant Microbe In. 2010;23:846-60.

18. Madhu B, Singh K, Saminathan M, Singh R, Shivasharanappa N, Sharma A, et al. Role of nitric oxide in the regulation of immune responses during rabies virus infection in mice. Virus disease. 2016;27:387-99.

19. Gupta KJ, Fernie AR, Kaiser WM, Dongen JT. On the origins of nitric oxide. Trends Plant Sci. 2011;16:160-8.

20. Crawford NM. Mechanisms for nitric oxide synthesis in plants. J Exp Bot 2006:57:471-8

21. Besson-Bard A, Pugin A, Wendehenne D. New insights into nitric oxide signaling in plants. The Annual Review of Plant Biology. 2008;59:21-39.

22. Kang K, Lee K, Park S, Byeon Y, Back K. Molecular cloning of rice serotonin $\mathrm{N}$-acetyltransferase, the penultimate gene in plant melatonin biosynthesis. J Pineal Res. 2013;55:7.

23. Tossi $V$, Lamattina L, Cassia R. An increase in the concentration of abscisic acid is critical for nitric oxide-mediated plant adaptive responses to UV-B irradiation. New Phytol. 2009;181:871-9.

24. Han B, Yang Z, Xie Y, Nie L, Cui J, Shen W. Arabidopsis HY1 confers cadmium tolerance by decreasing nitric oxide production and improving iron homeostasis. Mol Plant. 2013;7:388-403.

25. Zhou Y, Liu H, Wang G, Huang X, Cheng Z, Chen Z, et al. Immuno-detection of Rice stripe virus carried by small brown planthopper. Jiangsu Agric Sci. 2004;1:50-1.

26. Zhou T, Wang $Y$, Wu $\sqcup$, , Fan $Y$, Zhou $Y$. The research on method of artificial inoculation and identification on rice resistance to rice black dwarf virus. The Chinese society of plant pathology. 2011;04.

27. Balcerczyk A, Soszynski M, Bartosz G. On the specificity of 4-amino-5methylamino-2',,' '-difluorofluorescein as a probe for nitric oxide. Free Radic Biol Med. 2005:39:327-35.

28. Qi F, Xiang Z, Kou N, Cui W, Xu D, Wang R, et al. Nitric oxide is involved in methane-induced adventitious root formation in cucumber. Physiol Plant. 2017;159:366-77.

29. Fang $P, L u R$, Sun F, Lan $Y$, Shen W, Du L, et al. Assessment of reference gene stability in rice stripe virus and Rice black streaked dwarf virus infection rice by quantitative real-time PCR. Virol J. 2015;12:175-85.

30. Kenneth $J$, Schmittgen TD. Analysis of relative gene expression data using real-time quantitative PCR and the $2^{-\Delta \Delta C t}$ method. Methods. 2001;25:402-8.

31. Lozano-Juste J, León J. Enhanced abscisic acid-mediated responses in nia1nia2noa1-2 triple mutant impaired in NIA/NR- and AtNOA1-dependent nitric oxide biosynthesis in Arabidopsis. Plant Physiol. 2009;52:891-903.

32. Sanz L, Fernándezmarcos M, Modrego A, Lewis DR, Muday GK, Pollmann S, et al. Nitric oxide plays a role in stem cell niche homeostasis through its interaction with auxin. Plant Physiol. 2014;166:1972-84.

33. Shimono M, Sugano S, Nakayama A, Jiang CJ, Ono K, Toki S, et al. Rice WRKY45 plays a crucial role in benzothiadiazole-inducible blast resistance. Plant Cell. 2007;19:2064-76.

34. Nahar K, Kyndt T, Vleesschauwer DD, Höfte M, Gheysen G. The jasmonate pathway is a key player in systemically induced defense against root knot nematodes in rice. Plant Physiol. 2011;57:305-16.

35. Natarajan S, Luthria D, Bae H, Lakshman D, Mitra A. Transgenic soybeans and soybean protein analysis: an overview. J Agric Food Chem. 2013;61: $11736-43$

36. Zhao H, Xu L, Su T, Jiang Y, Hu L, Ma F. Melatonin regulates carbohydrate metabolism and defenses against Pseudomonas syringae pv. tomato DC3000 infection in Arabidopsis thaliana. J Pineal Res. 2015:59:109.

37. Kantar \$̧, Türközkan N, Bircan FS, Paşaoğlu ÖT. Beneficial effects of melatonin on serum nitric oxide, homocysteine, and ADMA levels in fructose-fed rats. Pharm Biol. 2015;53:1035-41.
38. Pozo D, Reiter R, Calvo J, Guerrero J. Physiological concentrations of melatonin inhibit nitric oxide synthase in rat cerebellum. Life Sci. 1994;55: 455-60.

39. Aydogan S, Yerer MB, Goktas A. Melatonin and nitric oxide. J Endocrinol Investig. 2006;29:281

40. Wang Q, Liu Y, He J, Zheng X, Hu J, Liu Y, et al. STV11 encodes a sulphotransferase and confers durable resistance to rice stripe virus. Nat Commun. 2014:5:4768.

41. Song F, Goodman RM. Activity of nitric oxide is dependent on, but is partially required for function of, salicylic acid in the signaling pathway in tobacco systemic acquired resistance. Mol Plant Microbe. 2001;14:1458-62.

42. Shimono M, Koga H, Akagi A, Hayashi N, Goto S, Sawada M, et al. Rice WRKY45 plays important roles in fungal and bacterial disease resistance. $\mathrm{Mo}$ Plant Pathol. 2012;13:83.

43. Yokotani N, Sato Y, Tanabe S, Chujo T, Shimizu T, Okada K, et al. WRKY76 is a rice transcriptional repressor playing opposite roles in blast disease resistance and cold stress tolerance. J Exp Bot. 2013;64:5085.

44. Qian Y, Tan D, Reiter R, Shi H. Comparative metabolomic analysis highlights the involvement of sugars and glycerol in melatonin-mediated innate immunity against bacterial pathogen in Arabidopsis. Sci Rep. 2015;5:15815.

\section{Publisher's Note}

Springer Nature remains neutral with regard to jurisdictional claims in published maps and institutional affiliations.
Ready to submit your research? Choose BMC and benefit from:

- fast, convenient online submission

- thorough peer review by experienced researchers in your field

- rapid publication on acceptance

- support for research data, including large and complex data types

- gold Open Access which fosters wider collaboration and increased citations

- maximum visibility for your research: over $100 \mathrm{M}$ website views per year

At $\mathrm{BMC}$, research is always in progress.

Learn more biomedcentral.com/submissions 